\title{
PET Demonstrates Functional Recovery After Transplantation of Induced Pluripotent Stem Cells in a Rat Model of Cerebral Ischemic Injury
}

\author{
Jiachuan Wang ${ }^{1-4}$, Fangfang Chao ${ }^{1-4}$, Feng Han ${ }^{5}$, Gensheng Zhang ${ }^{5}$, Qunying Xi ${ }^{1-4}$, Jinhui $\mathrm{Li}^{1-4}$, Han Jiang ${ }^{1-4}$, \\ Jing Wang ${ }^{1-4}$, Gang Yu ${ }^{1-4}$, Mei Tian ${ }^{1-4}$, and Hong Zhang ${ }^{1-4}$ \\ ${ }^{I}$ Department of Nuclear Medicine, Second Hospital of Zhejiang University School of Medicine, Hangzhou, China; ${ }^{2}$ Zhejiang \\ University Medical PET Center, Hangzhou, China; ${ }^{3}$ Institute of Nuclear Medicine and Molecular Imaging, Zhejiang University, \\ Hangzhou, China; ${ }^{4}$ Key Laboratory of Medical Molecular Imaging of Zhejiang Province, Hangzhou, China; and ${ }^{5}$ Institute of \\ Pharmacology, Toxicology, and Biochemical Pharmaceutics of Zhejiang University, Hangzhou, China
}

The purpose of this study was to determine the functionality of the transplanted induced pluripotent stem cells (iPSCs) and embryonic stem cells (ESCs) in a rat model of cerebral ischemia with use of ${ }^{18} \mathrm{~F}-\mathrm{FDG}$ small-animal PET imaging. Methods: Middle cerebral artery occlusion was used to establish cerebral ischemia. Twenty-four male rats were randomly assigned to 1 of 3 groups: iPSC treatment, ESC treatment, and the control phosphate-buffered saline (PBS) injection. After neurologic function tests and baseline ${ }^{18} \mathrm{~F}$-FDG small-animal PET had been performed, $1.0 \times$ $10^{6}$ suspended iPSCs or ESCs were injected stereotactically into the left lateral ventricle. The treatment response was evaluated weekly by ${ }^{18} \mathrm{~F}-\mathrm{FDG}$ PET scans and neurologic function tests. Histologic analyses and autoradiographic imaging were performed 4 wk after stem cell transplantation. Results: Compared with the PBS injection group, higher ${ }^{18} \mathrm{~F}-\mathrm{FDG}$ accumulation in the ipsilateral cerebral infarction was observed in both the iPSC and the ESC treatment groups during the 4 -wk period $(P<0.05)$. ${ }^{18} \mathrm{~F}-$ FDG accumulation in the ipsilateral cerebral infarction increased steadily over time in the iPSC treatment group. At 1 and 2 wk after stem cell transplantation, significant recovery of glucose metabolism was found in the ESC treatment group $(P<0.05)$ and then decreased gradually. The neurologic score in both stem celltreated groups was significantly lower than that in the PBS group, indicating functional improvement. Immunohistochemical analysis demonstrated that transplanted stem cells survived and migrated close to the ischemic region, and most of the stem cells expressed protein markers for cells of interest. Conclusion: ${ }^{18} \mathrm{~F}-\mathrm{FDG}$ small-animal PET demonstrated metabolic recovery after iPSC and ESC transplantation in the rat model of cerebral ischemia. iPSCs could be considered a potentially better therapeutic approach than ESCs and are worthy of further translational investigation.

Received Jul. 11, 2012; revision accepted Oct. 18, 2012. For correspondence or reprints contact either of the following: Hong Zhang, Department of Nuclear Medicine, Second Hospital of Zhejiang University, 88 Jiefang Rd., Hangzhou, Zhejiang 310009, China.

E-mail: hzhang21@gmail.com

Mei Tian, Department of Nuclear Medicine, Second Hospital of Zhejiang

University, 88 Jiefang Rd., Hangzhou, Zhejiang 310009, China.

E-mail: meitian@gmail.com

Published online Mar. 15, 2013.

COPYRIGHT (C) 2013 by the Society of Nuclear Medicine and Molecular Imaging, Inc.
Key Words: cerebral ischemia; induced pluripotent stem cells; transplantation; positron emission tomography (PET)

J Nucl Med 2013; 54:785-792

DOI: 10.2967/jnumed.112.111112

$\mathbf{I}$ schemic stroke is the second leading cause of death in industrialized countries (1). Current therapeutic strategies to improve neural function such as pharmacotherapy, interventional procedures, and surgery are limited because of the inability to replace damaged or lost neural cells. Recently, cell-based therapy using adult or embryonic stem cells (ESCs) has provided hope for enhancing tissue repair and functional recovery after ischemic stroke. In contrast to adult stem cells, which lack the potency to differentiate into multiple functional neural cell types, ESCs can give rise to many different types of cells of the whole body and hold the greatest potential for therapeutic applications. Over the past $10 \mathrm{y}$, although ESC therapy has been confirmed for the improvement of neural function in animal models $(2,3)$, its clinical application has been greatly limited because of the risk of immune rejection and ethical controversy. Hence, it is necessary to find an alternative source of pluripotent cells to replace ESCs.

Recently, induced pluripotent stem cells (iPSCs) were introduced as a new source of stem cells that can avoid the risk of immune rejection and ethical issues. iPSCs can be generated by transduction of defined transcription factors from adult somatic cells through reprogramming (4) and have been differentiated in vitro into the early neural stem cell stage or the neural lineage, including neurons and glial cells (5-7). More recently, iPSCs have been applied to a variety of nervous system disease models, including Parkinson disease (8), spinal cord injury $(9,10)$, and stroke $(11-14)$, and were found to improve neural function $(11,13,15)$. However, to better understand the in vivo behavior and efficacy of iPSCs, a noninvasive, sensitive, and clinically applicable 
approach for tracking the transplanted iPSCs and monitoring the therapeutic response in living subjects is warranted.

PET is one of the best-suited modalities to evaluate stem cell therapy, since it can be used in patients clinically for both cell trafficking and monitoring the response to therapy $(16,17)$. PET with ${ }^{18} \mathrm{~F}-\mathrm{FDG}$, a powerful tool with which to study the etiopathogenesis and progression of neurologic diseases, has recently been applied to detect the subtle changes of glucose metabolism in vivo after stem cell therapy in various neurologic disease models, including traumatic brain injury (18), Parkinson disease (19-21), and Huntington disease (22).

Although ESCs and iPSCs have been able to improve functional recovery in the compromised central nervous system $(18,21,22)$, no direct comparison had been made of these 2 different cell types in a single animal model of cerebral ischemia. Therefore, we wanted to directly compare the therapeutic effect of ESCs and iPSCs. For that, iPSCs and ESCs were transplanted into a rat model of cerebral ischemia, and the metabolic recovery of the cerebral infarction area was evaluated by ${ }^{18} \mathrm{~F}-\mathrm{FDG}$ small-animal PET, histologic analyses, and neurologic function tests.

\section{MATERIALS AND METHODS}

\section{Experimental Design and Animal Groups}

Each of 24 adult Sprague-Dawley male rats (body weight, 240-270 g) was randomly assigned to 1 of the following 3 experimental groups ( 8 per group): the iPSC transplantation group, the ESC transplantation group, and the phosphate-buffered saline (PBS) injection group. All experimental animals underwent middle cerebral artery occlusion (MCAO), and reperfusion was performed 90 min after MCAO. The stem cell transplantation or PBS injection was performed $3 \mathrm{~d}$ after reperfusion. Before stem cell transplantation and at weeks 1,2,3, and 4 after cell transplantation, all rats underwent neurologic function testing followed by ${ }^{18} \mathrm{~F}-\mathrm{FDG}$ small-animal PET scanning. Autoradiographic detection and immunohistochemical staining were done after the last ${ }^{18} \mathrm{~F}$-FDG smallanimal PET scan (Supplemental Fig. 1; supplemental materials are available online only at http://jnm.snmjournals.org).

All procedures associated with the care of animals were performed according to the National Institutes of Health Guide for the Care and Use of Laboratory Animals (23) and were approved by the Institutional Animal Care and Use Committee.

\section{Induction of Middle Cerebral Artery Occlusion}

Animal preparation and induction of anesthesia for the MCAO procedure and stem cell transplantation followed the institutional guidelines and the advice of veterinary staff. Briefly, after initial induction of anesthesia with a ketamine-xylazine mixture (a 60 $\mathrm{mg} / \mathrm{kg}$ dose of ketamine plus a $10 \mathrm{mg} / \mathrm{kg}$ dose of xylazine, intraperitoneally) (24), the rat was shaved, transported to the surgery table in a dedicated surgery room, and fixed on the platform with a warm pad (model 69001; RWD Life Science) to maintain the body temperature throughout the procedure. The animal received an intraperitoneal injection of $10 \%$ chloral hydrate $(350 \mathrm{mg} / \mathrm{kg})(24)$. Focal brain ischemia was induced by the intraluminal suture technique. The midline skin incision was made in the neck with subsequent exploration of the left common carotid artery, the external carotid artery, and the internal carotid artery. MCAO was achieved by advancing a 3-0 monofilament nylon suture with a rounded tip to the left internal carotid artery via the external carotid artery until slight resistance was felt. After 90 min of MCAO, reperfusion was performed by withdrawal of the suture until the tip cleared the internal carotid artery, and the wound was closed. The animals were allowed to recover until awake and were returned to their cages. Rats were treated for pain for the first $24 \mathrm{~h}$ after operation, by receiving an injection of buprenorphine $(0.05 \mathrm{mg} / \mathrm{kg}$, subcutaneously) every 8-12 h (25). More detailed postoperative care is described in the supplemental data. The rats were housed 3 per cage in environmentally enriched conditions under a 12-h light-dark cycle throughout the experiments and were allowed free access to food and water.

\section{Stem Cell Preparation}

Enhanced green fluorescent protein (EGFP)-labeled mouse iPSCs and ESCs were cultured as described previously (4). Briefly, mouse iPSCs and ESCs were maintained on a mitotically inactivated (mitomycin C) (Roche) mouse embryonic fibroblast feeder layer in knockout Dulbecco modified Eagle medium (Gibco) containing $10 \%$ fetal bovine serum (Hyclone), 10\% knockout serum replacement (Invitrogen), $2 \mathrm{mM}$ L-glutamine (Invitrogen), $\times 100$ nonessential amino acids (Invitrogen), $\times 1,000 \beta$-2-mercaptoethanol (Invitrogen), 50 units of penicillin and a $50 \mathrm{mg} / \mathrm{mL}$ dose of streptomycin (Invitrogen), and mouse leukemia inhibitory factor (Invitrogen). Before stem cell transplantation, iPSC and ESC colonies were passaged up to 4 times without feeder cells on $60-\mathrm{mm}$ culture dishes coated with $0.1 \%$ gelatin to eliminate contamination of the mouse embryonic fibroblasts.

\section{Transplantation Procedure}

After all the rats had been prepared, they were placed in a stereotactic instrument. A midline skin incision was made in the skull, with subsequent drilling for a burr hole. About $1.0 \times 10^{6}$ suspended iPSCs, ESCs, or PBS was stereotactically injected into the left lateral ventricle $(0.9 \mathrm{~mm}$ anterior to the bregma, $1.5 \mathrm{~mm}$ lateral to the midline, and $3.5 \mathrm{~mm}$ beneath the dura) in a volume of $20 \mu \mathrm{L}$ over $10 \mathrm{~min}$ with the use of a Hamilton microsyringe (26). The needle was left in place for an additional $10 \mathrm{~min}$ and then removed slowly. All surgical procedures were conducted under aseptic conditions. Postoperative pain control and care were performed as described in the section on the MCAO procedure.

\section{Neurologic Function Tests}

Testing of the Modified Neurologic Severity Score (mNSS) was performed before and after MCAO; at $3 \mathrm{~d}$ (immediately before stem cell transplantation); and at 1,2, 3, and 4 wk after cell transplantation by the same investigator, who was masked to the experimental groups, as described previously (27). Neurologic function was graded on a scale of $0-18(0=$ normal score; $18=$ maximal deficit score). The mNSS is a composite of motor, sensory, reflex, and balance tests. In the severity scores of injury, 1 score point is awarded for the inability to perform the test or for the lack of a tested reflex; thus, the higher the score, the more severe the injury.

\section{In Vivo PET Study and Image Analysis}

At $3 \mathrm{~d}$ after reperfusion and at 1,2,3, and $4 \mathrm{wk}$ after stem cell transplantation, rats were anesthetized with isoflurane (2\%) and injected with approximately $18.5 \mathrm{MBq}(500 \mu \mathrm{Ci})$ of ${ }^{18} \mathrm{~F}-\mathrm{FDG}$ via the tail vein. At $40 \mathrm{~min}$ after ${ }^{18} \mathrm{~F}$-FDG injection, the rats were anesthetized with isoflurane $(2 \%)$ and positioned prone in the microPET R4 scanner (Siemens Medical Solutions) for a 10-min static acquisition with the mid skull in the center of the field of 
view. The images were reconstructed with use of a modified backprojection algorithm. ${ }^{18} \mathrm{~F}$-FDG uptake was calculated as the percentage injected dose per gram of tissue using the AMIDE software package (version 9.2; Stanford University). In each image plane, 3-dimensional regions of interest $2 \mathrm{~mm}$ in diameter were drawn over the infarcted area of the cerebral cortex and the contralateral normal tissue, and the mean percentage injected dose per gram of tissue was averaged. The lesion-to-normal homologous contralateral (L/N) ratio was used for semiquantitative analysis. The $\mathrm{L} / \mathrm{N}$ ratio was calculated with use of the following formula: $\mathrm{L} / \mathrm{N}$ ratio $=$ mean counts per pixel of lesion region of interest/mean counts per pixel of contralateral normal area. The average radioactivity concentration within the infarcted area was obtained from the mean pixel values, normalized to that of nonischemic cortex, and expressed as a percentage.

\section{Autoradiographic Imaging}

Immediately after the last small-animal PET study, 6 animals ( 2 per group) were decapitated under deep anesthesia; the brains were removed rapidly into a rodent brain matrix and placed into the cryostat (Bright Instrument Co. Ltd.) for about $60 \mathrm{~min}$ at $-20^{\circ} \mathrm{C}$. Coronal brain slices (1-mm thick) were cut and kept in contact with an imaging plate (BAS-IP MS 2040; Fujifilm) for $20 \mathrm{~min}$. The exposed plates were scanned with a bioimaging analyzer (FLA-7000; Fujifilm), and the images were displayed with a Dell computer (Image Reader FLA-7000, version 1.12; Fujifilm) for qualitative evaluation.

\section{Immunohistochemical and Immunofluorescent Staining}

After the last small-animal PET scan, 18 animals (6 per group) were transcardially perfused with saline followed by $200 \mathrm{~mL}$ of 4\% paraformaldehyde in phosphate buffer under deep anesthesia (supplemental data). The brains were dissected and fixed in paraformaldehyde for 6-8 $\mathrm{h}$ and then dehydrated with $30 \%$ sucrose in $0.1 \mathrm{M}$ PBS for $2 \mathrm{~d}$. The brains were frozen on powdered dry ice, and then coronal sections ( $20 \mu \mathrm{m}$ thick) were processed with a cryostat (Leica) at $20^{\circ} \mathrm{C}$ throughout the entire ischemic damaged brain.

For immunohistochemical staining, the sections were permeabilized with $0.04 \%$ Triton X-100 and blocked with $10 \%$ normal goat serum and $0.5 \%$ bovine serum albumin in PBS for $1 \mathrm{~h}$ and then treated overnight at $4{ }^{\circ} \mathrm{C}$ with primary antibodies: Rabbit polyclonal antibody against glial fibrillary acidic protein (GFAP) (1:300 dilution; Abcam), mouse monoclonal antibody against neuronal nuclei (NeuN) (1:200 dilution; clone: A60, Millipore), or rabbit polyclonal antibody against von Willebrand factor (vWF) (1:200 dilution; DAKO). The sections were washed 3 times in PBS for 10 min each and incubated in biotinylated secondary antiserum (Vectastain $\mathrm{ABC}$ kit, Vector Lab) for $30 \mathrm{~min}$ at room temperature. The sections were rinsed and incubated in $\mathrm{ABC}$ reagents (Vectastain $\mathrm{ABC}$ kit) for $30 \mathrm{~min}$ and then washed thoroughly and incubated in $0.05 \%$ diaminobenzidine (DAB) and $0.03 \% \mathrm{H}_{2} \mathrm{O}_{2}$ for $3-5$ min until a brown reaction product was observed.

Immunohistochemical studies were performed to determine whether transplanted stem cells can induce neurogenesis and angiogenesis. NeuN was used as a mature neuronal marker, GFAP as the mature astrocyte marker, and vWF as the endothelial cell marker. We counted the number of cells positively stained with NeuN or vWF and calculated the mean values and SDs. The number of positively stained cells with NeuN or vWF in 3 different microscopic fields $(\times 200$ magnification) was calculated. To evaluate the average integral optical density (IOD) of GFAP, the hot spots of the section were selected with the use of a microscope $(\times 100$ magnification). Individual counts were then made in $\times 200$ fields (BX60; Olympus). Sections (3 microscopic fields per slice, 1 slice per animal) were digitized with the use of a camera. The IOD of GFAP was obtained using Image-ProPlus 5.0 software (Media Cybernetics).

To follow up on the fate of EGFP-labeled transplanted cells, some sections were processed for immunofluorescent analysis of the cell implants. Sections were blocked and incubated overnight at $4^{\circ} \mathrm{C}$ with primary antibodies as mentioned above; the dilution of all primary antibodies was 1:100. After washes in PBS, sections were incubated with fluorescence-conjugated secondary antibodies (Alexa Fluor 568, 1:500; Invitrogen) for $1 \mathrm{~h}$ at room temperature. Sections were washed and counterstained with the nuclear dye 4,6diamino-2-phenylindole. Fluorescence-labeled sections were viewed with use of a confocal microscope (Olympus).

\section{Statistical Analyses}

The data were expressed as mean \pm SD. A difference between groups was considered statistically significant if the $P$ value was less than 0.05 . The Student $t$ test was used to compare body weight between treatment group and control group after MCAO. ANOVA was used when the groups numbered 3. Statistical analyses were performed with SPSS software (version 15.0, SPSS Inc.).

\section{RESULTS}

\section{Neurologic Assessment After iPSC and ESC Transplantation}

To determine whether the transplantation of iPSCs and ESCs can improve sensorimotor deficit in ischemic brain injury, neurologic tests were performed and compared with each other by the same investigator, who was masked to the experimental groups ( 8 per group at each time point). No significant differences in mNSS scores were detected among the 3 experimental groups at $3 \mathrm{~d}$ after reperfusion (immediately before stem cell transplantation). The mNSS scores in the iPSC and ESC groups, starting $1 \mathrm{wk}$ after reperfusion, were significantly lower at each time point than were those of the PBS group $(P<0.05)$. At each time point, no significant differences in mNSS scores were detected between the iPSC group and the ESC group $(P>0.05)$. These results suggested that transplantation of iPSCs and ESCs improved neurologic function recovery (Fig. 1).

\section{F-FDG PET Evaluation}

To document that a stem cell implant corresponds to enhanced glucose metabolic activity, the rats were studied by ${ }^{18}$ F-FDG small-animal PET. The ${ }^{18}$ F-FDG PET scans allowed the visualization and quantification of glucose metabolism throughout the brain at each time point (Fig. 2A). Semiquantitative measurement of ${ }^{18} \mathrm{~F}-\mathrm{FDG}$ radioactivity in the ischemic area demonstrated no significant differences in the $\mathrm{L} / \mathrm{N}$ ratio among the 3 experimental groups at $3 \mathrm{~d}$ after reperfusion (immediately before stem cell transplantation) (Fig. 2B). However, the $\mathrm{L} / \mathrm{N}$ ratios were significantly increased in both the iPSC-treated and the ESC-treated groups at 1, 2, 3, and $4 \mathrm{wk}$ after stem cell transplantation $(P<0.001)$, indicating that transplantation of iPSCs and ESCs increased glucose metabolism in the cerebral ischemic 


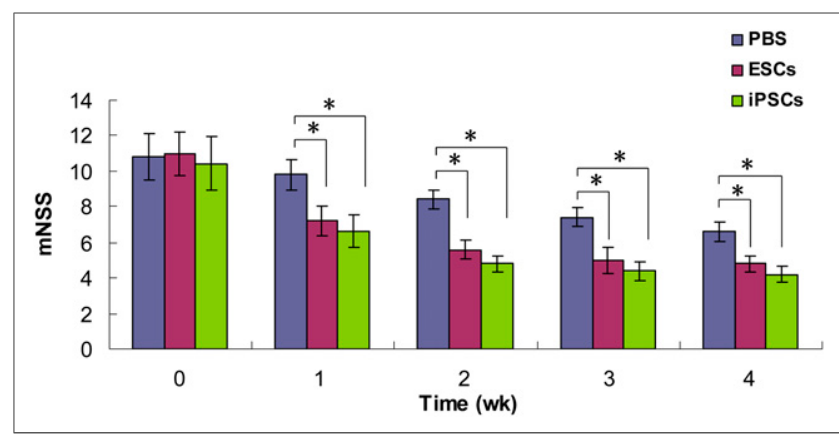

FIGURE 1. mNSS indicating neurologic function before and after stem cell transplantation. Groups are as follows: PBS (MCAO with PBS injection); ESCs (MCAO with ESC transplantation); and iPSCs (MCAO with iPSC transplantation at $3 \mathrm{~d}$ after reperfusion). Significant stem cell effects were detected with use of mNSS test at each time point, starting $1 \mathrm{wk}$ after treatment; ${ }^{*} P<0.05$.

area. Furthermore, in the ESC-treated group, the L/N ratio increased the most at $1 \mathrm{wk}$ after transplantation and was significantly higher at that time than that of the iPSC-treated group $(P<0.05)$; after that, the ratio decreased gradually until it became lower than that of the iPSC-treated group at $4 \mathrm{wk}$ after transplantation $(P<0.05)$. At $3 \mathrm{wk}$ after stem cell transplantation, no significant difference was found between the ESC-treated group and the iPSC-treated group $(P=0.97)$.

In addition, there was no focal abnormal increase in glucose metabolism in the cerebral ischemic area, thus indicating no tumor or teratoma formation at $4 \mathrm{wk}$ after stem cell transplantation.

\section{Autoradiographic Imaging}

The autoradiographic results demonstrated that the level of ${ }^{18} \mathrm{~F}-\mathrm{FDG}$ radioactivity detected was similar to that detected by ${ }^{18} \mathrm{~F}-\mathrm{FDG}$ small-animal PET in the same brain regions, as expected. Also as expected, activity distribution was similar but not identical (because of technical and physical differences) (Fig. 3), indicating a significant correlation between in vivo small-animal PET images and in vitro autoradiographic measurements.

\section{Immunohistochemical Findings}

Immunohistochemical studies were performed to determine whether transplanted stem cells can induce neurogenesis and angiogenesis. NeuN was used as a mature neuronal marker, GFAP as the mature astrocyte marker, and vWF as the endothelial cell marker. We counted the number of NeuN or vWF cells immunostaining positively and calculated the mean values and SDs. In the iPSC-treated animals, the number of NeuN-positive cells was significantly higher than that in the PBS-injected group $(56.5 \pm 13.88$ vs. $36.75 \pm$ 2.06; $P<0.05)$ and was higher than that in the ESC-treated group, but not to a statistically significant extent $(56.5 \pm$ 13.88 vs. $45.88 \pm 7.64 ; P=0.138$ ). There was no statistically significant difference between the ESC-treated group and the PBS-injected group (45.88 \pm 7.64 vs. $36.75 \pm 2.06$;

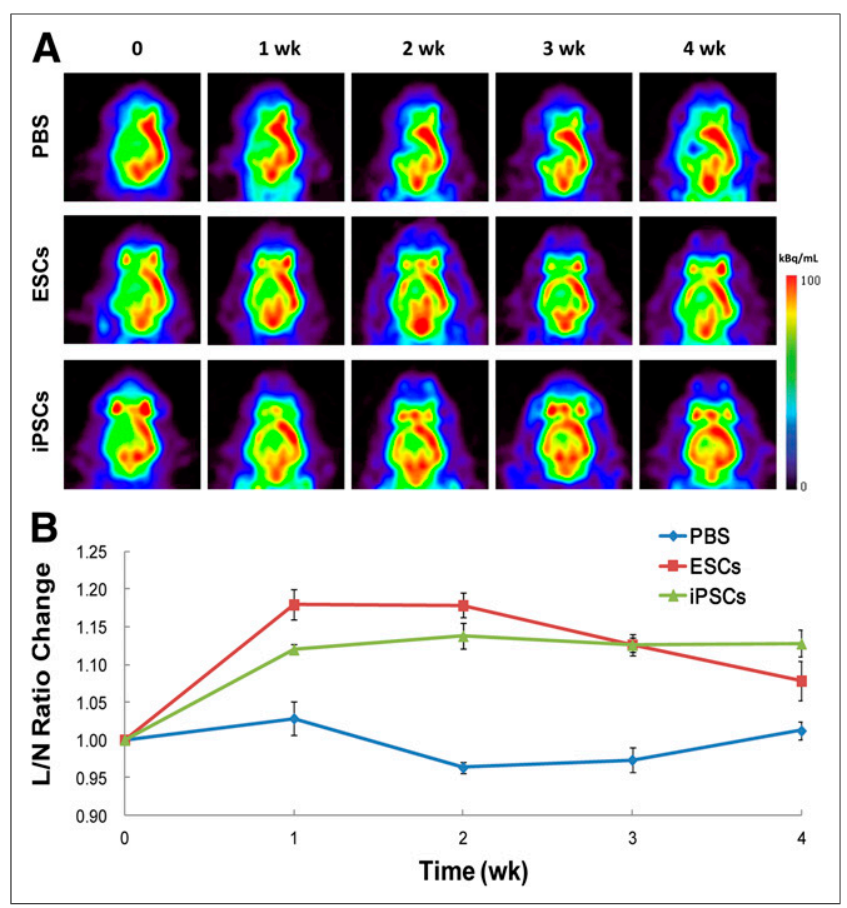

FIGURE 2. (A) Serial PET images demonstrating metabolism recovery after stem cell treatment for MCAO in rats. The 3 groups are presented as PBS (MCAO with PBS injection); ESCs (MCAO with ESC transplantation); and iPSCs (MCAO with iPSC transplantation). Images are shown in axial view. Scale was set according to signal intensity. (B) Semiquantitative analysis of variance of glucose metabolism after stem cell transplantation in each group (shown as change of $\mathrm{L} / \mathrm{N}$ ratio at each time point after stem cell transplantation relative to ratio of $\mathrm{L} / \mathrm{N}$ before transplantation). $\% \mathrm{ID}=$ percentage injected dose.

$P=0.195$ ) (Fig. 4). The number of vWF-positive cells in both the iPSC-treated group and the ESC-treated group was significantly higher than that in the PBS-injected group $(10.5 \pm 1.29$ vs. $7.38 \pm 1.38[P=0.022]$ and $10 \pm 1.31$ vs. $7.38 \pm 1.38[P=0.046]$, respectively). However, there was no statistically significant difference between the iPSC-treated group and the ESC-treated group (10.5 \pm 1.29 vs. $10 \pm 1.31 ; P=0.67$ ) (Fig. 5).

The IOD of GFAP in the iPSC-transplanted group was significantly higher than that in either the ESC-transplanted group $(4,552.25 \pm 401.85$ vs. $3,322.59 \pm 352.73 ; P=$ $0.001)$ or the PBS-injected group $(4,552.25 \pm 401.85$ vs. $2,600.91 \pm 363.04 ; P<0.001)$. Meanwhile, the IOD of GFAP in the ESC-transplanted group was significantly higher than that of the PBS-injected group $(3,322.59 \pm$ 352.73 vs. $2,600.91 \pm 363.04 ; P=0.023$ ) (Fig. 6).

Four weeks after transplantation, tracing of EGFPlabeled cells showed that the transplanted cells survived and integrated into the area close to the infarction. Double labeling of EGFP with NeuN, GFAP, or vWF showed that most of the grafted cells were $\mathrm{NeuN}^{+}, \mathrm{GFAP}^{+}$, or $\mathrm{vWF}^{+}$, which indicated the differentiation of iPSCs and ESCs into mature nerve cells (astrocytes and neurons) and angiogenic cells in vivo (Fig. 7). 


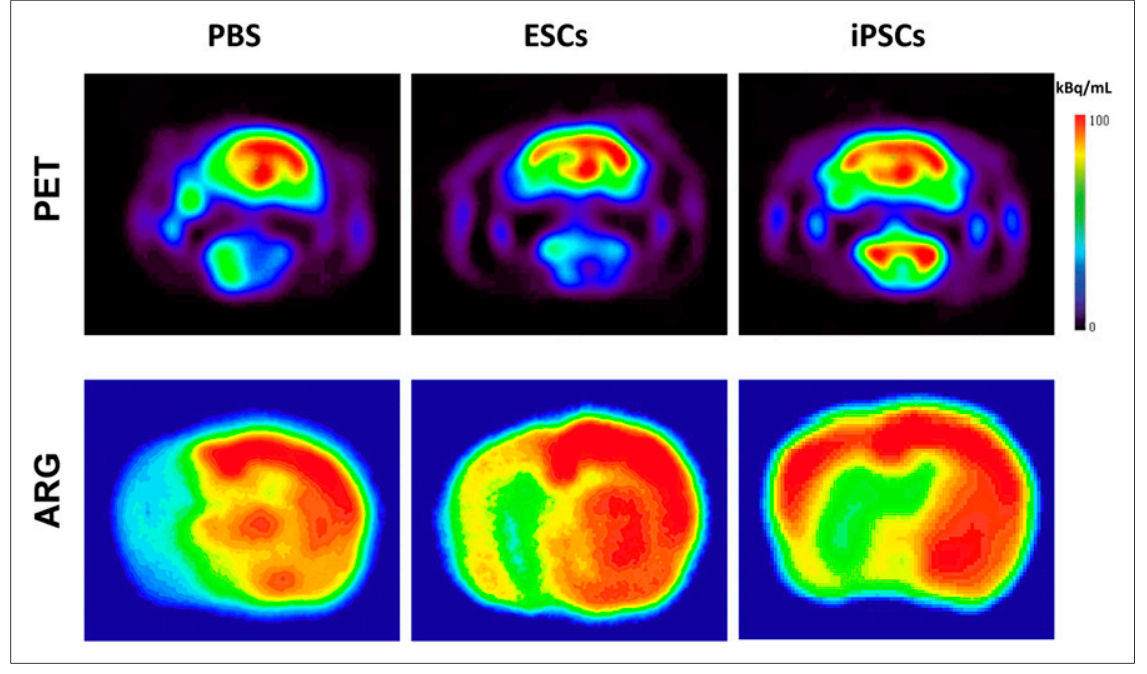

FIGURE 3. Transaxial slices of autoradiographic imaging together with PET scan. Scale was set according to signal intensity. $A R G=$ autoradiography.
Moreover, we did not observe nonneuronal or noncortical ${ }^{18}$ F-FDG-avid tissue formation (tumor or teratoma) in the transplant recipients at $4 \mathrm{wk}$ after iPSC and ESC transplantation.

\section{DISCUSSION}

In this study, the potential therapeutic effects of iPSCs and ESCs were evaluated and compared by serial ${ }^{18} \mathrm{~F}-\mathrm{FDG}$ small-animal PET scans combined with 2 other complementary approaches: neurologic function testing and immunohistochemical examination. To the best of our knowledge, this was the first study to use serial ${ }^{18} \mathrm{~F}$-FDG PET to evaluate the metabolic recovery of cerebral ischemia in rats after iPSC treatment. By using a rat model of cerebral ischemia, we were able to find increased glucose metabolic activity in the cerebral infarction area and improved neurologic function during the 4-wk period after iPSC and ESC transplantation. Immunohistologic analysis also confirmed the excellent

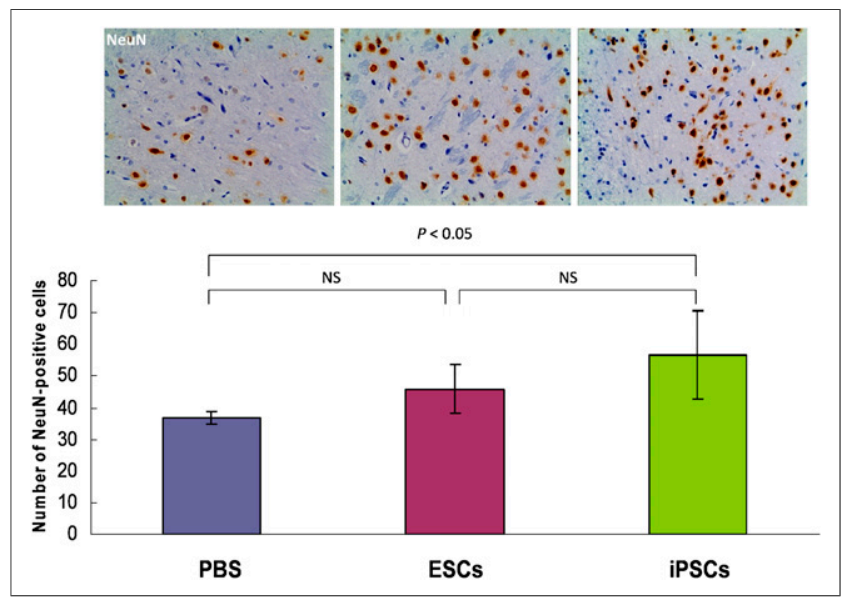

FIGURE 4. Photomicrograph $(\times 200)$ of immunohistochemical analysis of NeuN-positive cells (brown cells) in cerebral ischemic region in iPSC-treated and ESC-treated animals or PBS-injected animals. Number of NeuN-positive cells was significantly higher in iPSC-transplanted group. ARG = autoradiography. survival of iPSCs and ESCs in these animals at 4 wk after stem cell transplantation. We observed that iPSCs and ESCs migrated to the area close to the infarction, where they expressed the mature neuronal marker $\mathrm{NeuN}$, the mature astrocyte marker GFAP, and the endothelial cell marker vWF.

In this study, serial ${ }^{18} \mathrm{~F}-\mathrm{FDG}$ PET was used to evaluate cerebral glucose metabolism after iPSC and ESC treatment. Compared with the PBS control group, the higher ${ }^{18} \mathrm{~F}-\mathrm{FDG}$ accumulation in the ipsilateral cerebral infarction was observed in both the iPSC and the ESC treatment groups at 1-4 wk after stem cell transplantation. In earlier stages (at 1 and 2 wk after stem cell transplantation), significantly better metabolic recovery was found in the ESC treatment group, but in later stages (from $3 \mathrm{wk}$ after transplantation), the iPSC treatment group had higher ${ }^{18} \mathrm{~F}-\mathrm{FDG}$ accumulation than did the ESC group. These results indicated that iPSCs and ESCs transplanted into the brain might have improved the rats' recovery from experimental cerebral ischemia. Most

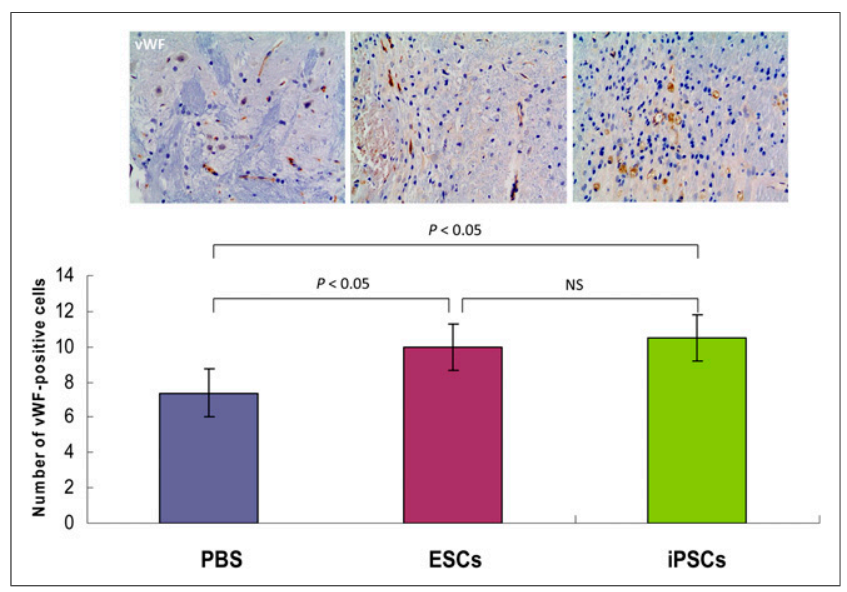

FIGURE 5. Photomicrograph $(\times 200)$ of immunohistochemical analysis of vWF-positive cells (brown cells) in cerebral ischemic region in iPSC-treated and ESC-treated animals or PBS-injected animals. Number of VWF-positive cells was significantly higher in both iPSC-transplanted and ESC-treated groups. 


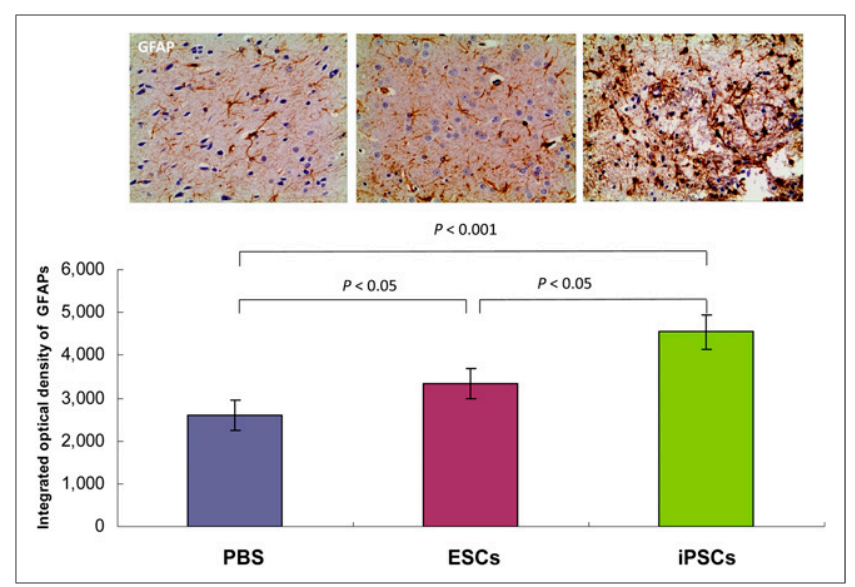

FIGURE 6. Photomicrograph of immunohistochemical analysis of GFAP-positive cells (brown cells) in cerebral ischemic region in iPSC-treated and ESC-treated animals or PBS-injected animals. IOD of GFAP was significantly higher in both iPSC-transplanted and ESC-treated groups. IOD of GFAP was also higher in iPSCtransplanted animals than in ESC-treated animals.

interestingly, the iPSC-treated group demonstrated steadily increasing ${ }^{18} \mathrm{~F}-\mathrm{FDG}$ accumulation during the 4-wk period, whereas the ESC group showed higher accumulation only in the earlier stage, indicating that iPSC treatment may produce a more stable therapeutic response than that of the ESC group.

What are the mechanisms or factors that promote reduced deficits with iPSC and ESC transplantation after stroke? One possibility is that the transplanted stem cells integrate into the tissue, replace damaged cells, and reconstruct the neural circuitry. In the present study, immunohistochemical analyses indicated that many transplanted cells survived and integrated close to the ischemic region and that most of the cells expressed protein markers for parenchymal cells. Quantitative analyses revealed that the numbers of neurons, astrocytes, and endothelial cells were higher in the iPSC treatment group than in the ESC treatment group, as was consistent with the recovery of glucose metabolism in cerebral infarction evaluated by our ${ }^{18} \mathrm{~F}$-FDG PET scan. These changes indicate that iPSC transplantation-induced neurogenesis and angiogenesis play an important role during recovery.

Another reasonable explanation is that changes in the microenvironment may contribute to tissue protection and repair (28) or that the interaction of grafted stem cells with the host brain may lead to production of trophic factors (29). Previous reports demonstrated that transplantation of human microglial cells into the ischemic brain induced a significant upregulation in expression of glial cell linederived neurotrophic factor and vascular endothelial growth factor in the ischemic penumbra (30). The presence of upregulated glial cell line-derived neurotrophic factor and vascular endothelial growth factor in the rat brain might be an explanation for the neuronal recovery because those factors can support neuronal survival and attenuate neuronal apoptotic death (31).

The reason for the neuronal recovery remains elusive, but various factors including the apoptotic signal pathway, necrosis, and the repair process may affect it. A few studies have reported that transplantation of gene-modified stem cells overexpressing glial cell line-derived neurotrophic factor, brain-derived neurotrophic factor, or vascular endothelial growth factor after cerebral ischemia reduced infarct volume and ameliorated neurologic deficits (32-34). However, whether the iPSCs and ESCs themselves function in this way, or whether their differentiated cells have the corresponding functions, needs to be further investigated.

Despite the clear advantages of pursuing iPSC technology, ESCs remain the only pluripotent cells that can be considered genetically unmodified and still hold the greatest potential for useful therapeutic applications. This is why we used ESCs as the gold standard in terms of pluripotency in the present study. In addition, there are several similar properties between iPSCs and ESCs, such as their morphology, self-renewal, differentiation capacity, and cell surface antigens (35).

However, there are still differences between the 2 cell types, such as their gene expression profiles. Studies have identified more than 1,200 gene differences between iPSCs and ESCs (36). Another report demonstrated that residual gene expression of the donor cell type contributes significantly to the differences between human iPSCs and human ESCs (37). The time course during neuroectodermal differentiation was also different in iPSCs and ESCs. For example, 2 recent studies compared the capacity of human iPSCs and human ESCs to differentiate into neural cells (neurons and glia) and found that iPSC-derived neural cells had greater variation and lower efficiency in neuronal differentiation. However, once iPSCs were specified to a neural lineage, they could be differentiated to form motor neurons with efficiency similar to that of human ESCs $(6,38)$.

Until now, only a few studies have directly compared the efficacy of treatment for cerebral ischemia between iPSCs and ESCs. In the present study, we directly compared these 2 types of stem cells and found differences in the recovery of glucose metabolism and in neurogenesis and angiogenesis that were induced by iPSC and ESC transplantation. However, whether the metabolic and functional changes observed in this study resulted from the neurologic differentiation in vivo between iPSCs and ESCs in the different time courses remains unclear and needs to be determined in future studies.

The present study had several limitations. One was that the study was performed for only 4 wk after stem cell transplantation. We therefore could not form conclusions about longer-term therapeutic response after iPSC transplantation, including potential tumor formation, although we did not find any tumor formation in this study. The second limitation is that we did not use the direct cell labeling or transgenic cell labeling technique to track the transplanted stem cells. However, direct monitoring of metabolic recovery by ${ }^{18} \mathrm{~F}$-FDG PET did have certain advantages. For example, it did not interfere with the normal stem cell potential for life, and this technique can easily be translated and applied 


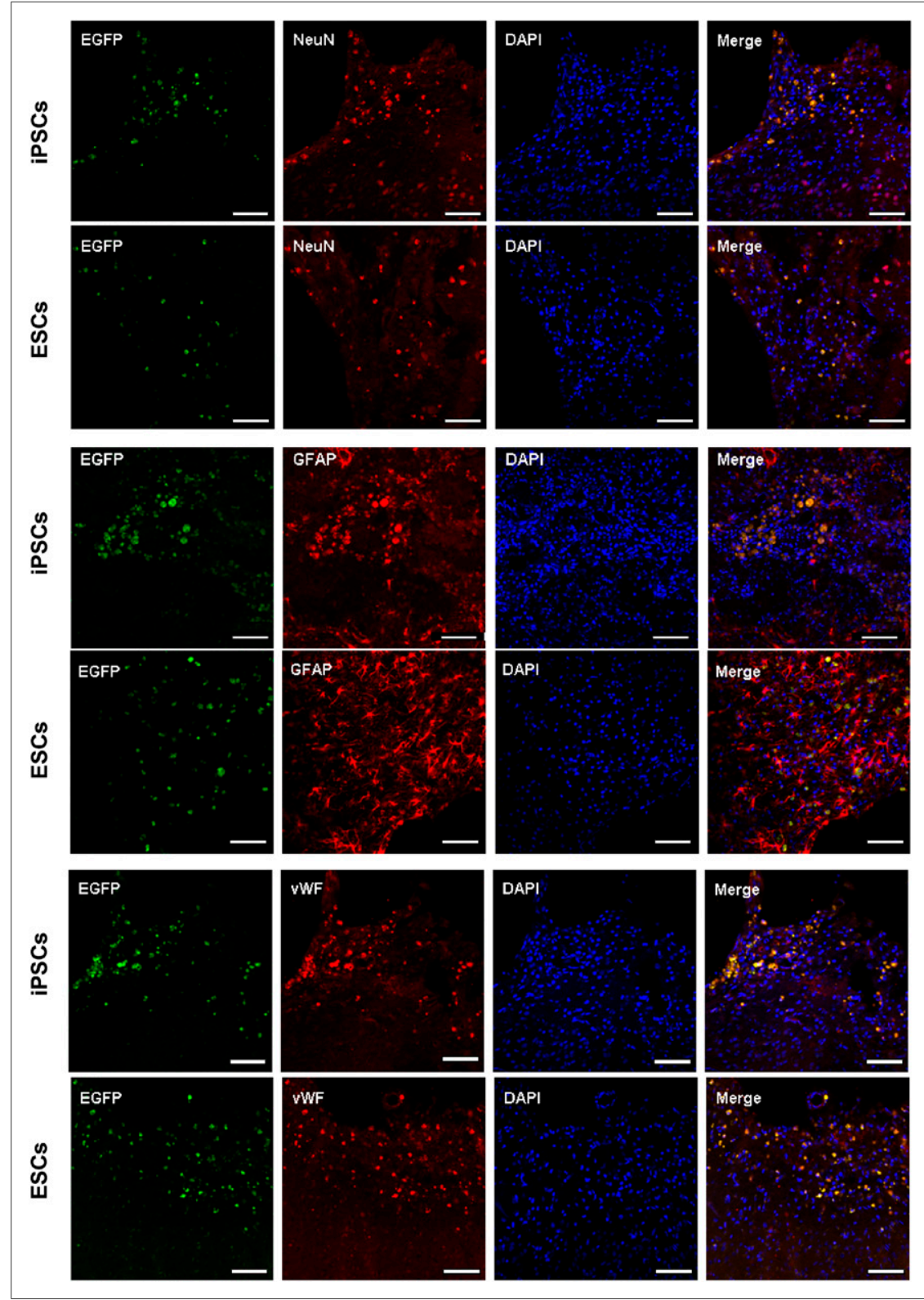

FIGURE 7. Photomicrographs ( $\times 200)$ showing differentiation of transplanted stem cells in brain of rats with cerebral ischemia 4 wk after transplantation. Transplanted stem cells show green fluorescence; immunostaining with antibodies against NeuN (mature neuron markers), GFAP (astrocyte markers), and VWF (endothelial cell markers) show red fluorescence; nuclei stained with 4,6diamino-2-phenylindole show blue fluorescence; and merged images show that engrafted iPSCs and ESCs express neurons, astrocytes, or endothelial cell features. Scale bar $=50 \mu \mathrm{m}$. widely in clinical practice. The third limitation is that complete kinetic modeling has not been performed to confirm the true dynamic of glucose metabolism after injection of ${ }^{18} \mathrm{~F}$-FDG. This modeling should be conducted in the further investigations.

\section{CONCLUSION}

Serial ${ }^{18}$ F-FDG small-animal PET demonstrated metabolic recovery after iPSC and ESC transplantation in a rat model of cerebral ischemia. iPSCs can be considered a potentially better therapeutic approach than ESCs and are worthy of further translational investigations.

\section{DISCLOSURE}

The costs of publication of this article were defrayed in part by the payment of page charges. Therefore, and solely to indicate this fact, this article is hereby marked "advertisement" in accordance with 18 USC section 1734. This work was partly sponsored by grants from the Zhejiang Provincial Natural Science Foundation of China (Z2110230), the Health Bureau of Zhejiang Province (2010ZA075, 2011ZDA013), the Science and Technology Bureau of Zhejiang Province (2012R10040), the China Postdoctoral Science Foundation (20110491791), the National Science Foundation of China (NSFC) $(81101023,81173468,81271601)$, and the Ministry of Science and Technology of China (2011CB504400, 2012BAI13B06). No other potential conflict of interest relevant to this article was reported.

\section{ACKNOWLEDGMENT}

We thank Tamara K. Locke for her excellent scientific editing of the manuscript. 


\section{REFERENCES}

1. Kleinschnitz C, Grund H, Wingler K, et al. Post-stroke inhibition of induced NADPH oxidase type 4 prevents oxidative stress and neurodegeneration. PLoS Biol. 2010;8:e1000479.

2. Theus $\mathrm{MH}$, Wei $\mathrm{L}$, Cui $\mathrm{L}$, et al. In vitro hypoxic preconditioning of embryonic stem cells as a strategy of promoting cell survival and functional benefits after transplantation into the ischemic rat brain. Exp Neurol. 2008;210:656-670.

3. Wei L, Cui L, Snider BJ, et al. Transplantation of embryonic stem cells overexpressing Bcl-2 promotes functional recovery after transient cerebral ischemia. Neurobiol Dis. 2005;19:183-193.

4. Takahashi K, Yamanaka S. Induction of pluripotent stem cells from mouse embryonic and adult fibroblast cultures by defined factors. Cell. 2006;126:663-676.

5. Jensen MB, Yan H, Krishnaney-Davison R, Al Sawaf A, Zhang SC. Survival and differentiation of transplanted neural stem cells derived from human induced pluripotent stem cells in a rat stroke model. J Stroke Cerebrovasc Dis. November 10, 2011 [Epub ahead of print].

6. Karumbayaram S, Novitch BG, Patterson M, et al. Directed differentiation of human-induced pluripotent stem cells generates active motor neurons. Stem Cells. 2009;27:806-811.

7. Kitazawa A, Shimizu N. Differentiation of mouse induced pluripotent stem cells into neurons using conditioned medium of dorsal root ganglia. $N$ Biotechnol. 2011;28:326-333.

8. Hargus G, Cooper O, Deleidi M, et al. Differentiated Parkinson patient-derived induced pluripotent stem cells grow in the adult rodent brain and reduce motor asymmetry in Parkinsonian rats. Proc Natl Acad Sci U S A. 2010;107:15921-15926.

9. Tsuji O, Miura K, Okada Y, et al. Therapeutic potential of appropriately evaluated safe-induced pluripotent stem cells for spinal cord injury. Proc Natl Acad Sci U S A. 2010;107:12704-12709.

10. Nori S, Okada Y, Yasuda A, et al. Grafted human-induced pluripotent stem-cellderived neurospheres promote motor functional recovery after spinal cord injury in mice. Proc Natl Acad Sci U S A. 2011;108:16825-16830.

11. Jiang M, Lv L, Ji H, et al. Induction of pluripotent stem cells transplantation therapy for ischemic stroke. Mol Cell Biochem. 2011;354:67-75.

12. Yamashita T, Kawai H, Tian F, Ohta Y, Abe K. Tumorigenic development of induced pluripotent stem cells in ischemic mouse brain. Cell Transplant. 2011; 20:883-891.

13. Chen SJ, Chang CM, Tsai SK, et al. Functional improvement of focal cerebral ischemia injury by subdural transplantation of induced pluripotent stem cells with fibrin glue. Stem Cells Dev. 2010;19:1757-1767.

14. Kawai H, Yamashita T, Ohta Y, et al. Tridermal tumorigenesis of induced pluripotent stem cells transplanted in ischemic brain. J Cereb Blood Flow Metab. 2010;30:1487-1493.

15. Cai J, Yang M, Poremsky E, Kidd S, Schneider JS, Iacovitti L. Dopaminergic neurons derived from human induced pluripotent stem cells survive and integrate into 6-OHDA-lesioned rats. Stem Cells Dev. 2010;19:1017-1023.

16. Zhang Y, Ruel M, Beanlands RS, deKemp RA, Suuronen EJ, DaSilva JN. Tracking stem cell therapy in the myocardium: applications of positron emission tomography. Curr Pharm Des. 2008;14:3835-3853.

17. Jiang $\mathrm{H}$, Cheng $\mathrm{Z}$, Tian $\mathrm{M}$, Zhang $\mathrm{H}$. In vivo imaging of embryonic stem cell therapy. Eur J Nucl Med Mol Imaging. 2011;38:774-784.

18. Zhang $\mathrm{H}$, Zheng $\mathrm{X}$, Yang $\mathrm{X}$, et al. ${ }^{11} \mathrm{C}-\mathrm{NMSP} /{ }^{18} \mathrm{~F}-\mathrm{FDG}$ microPET to monitor neural stem cell transplantation in a rat model of traumatic brain injury. Eur J Nucl Med Mol Imaging. 2008;35:1699-1708.
19. Muramatsu S, Okuno T, Suzuki Y, et al. Multitracer assessment of dopamine function after transplantation of embryonic stem cell-derived neural stem cells in a primate model of Parkinson's disease. Synapse. 2009;63:541-548.

20. Jackson J, Chapon C, Jones W, Hirani E, Qassim A, Bhakoo K. In vivo multimodal imaging of stem cell transplantation in a rodent model of Parkinson's disease. J Neurosci Methods. 2009;183:141-148.

21. Shyu WC, Li KW, Peng HF, et al. Induction of GAP-43 modulates neuroplasticity in PBSC (CD34+) implanted-Parkinson's model. J Neurosci Res. 2009;87: 2020-2033.

22. Visnyei K, Tatsukawa KJ, Erickson RI, et al. Neural progenitor implantation restores metabolic deficits in the brain following striatal quinolinic acid lesion. Exp Neurol. 2006;197:465-474.

23. Guide for the Care and Use of Laboratory Animals. 8th ed. Washington, DC: National Academy Press; 2011.

24. Zhu M, Ackerman JJ, Yablonskiy DA. Body and brain temperature coupling: the critical role of cerebral blood flow. J Comp Physiol B. 2009;179:701-710.

25. Curtin LI, Grakowsky JA, Suarez M, et al. Evaluation of buprenorphine in a postoperative pain model in rats. Comp Med. 2009;59:60-71.

26. Sugiura S, Kitagawa K, Tanaka S, et al. Adenovirus-mediated gene transfer of heparin-binding epidermal growth factor-like growth factor enhances neurogenesis and angiogenesis after focal cerebral ischemia in rats. Stroke. 2005;36:859-864.

27. Chen J, Sanberg PR, Li Y, et al. Intravenous administration of human umbilical cord blood reduces behavioral deficits after stroke in rats. Stroke. 2001;32:26822688.

28. Feng M, Zhu H, Zhu Z, et al. Serial ${ }^{18}$ F-FDG PET demonstrates benefit of human mesenchymal stem cells in treatment of intracerebral hematoma: a translational study in a primate model. J Nucl Med. 2011;52:90-97.

29. Sadan O, Shemesh N, Barzilay R, et al. Mesenchymal stem cells induced to secrete neurotrophic factors attenuate quinolinic acid toxicity: a potential therapy for Huntington's disease. Exp Neurol. 2012;234:417-427.

30. Narantuya D, Nagai A, Sheikh AM, et al. Human microglia transplanted in rat focal ischemia brain induce neuroprotection and behavioral improvement. PLoS ONE. 2010;5:e11746.

31. Kilic U, Kilic E, Dietz GP, Bahr M. Intravenous TAT-GDNF is protective after focal cerebral ischemia in mice. Stroke. 2003;34:1304-1310.

32. Horita Y, Honmou O, Harada K, Houkin K, Hamada H, Kocsis JD. Intravenous administration of glial cell line-derived neurotrophic factor gene-modified human mesenchymal stem cells protects against injury in a cerebral ischemia model in the adult rat. J Neurosci Res. 2006;84:1495-1504.

33. Zhu JM, Zhao YY, Chen SD, Zhang WH, Lou L, Jin X. Functional recovery after transplantation of neural stem cells modified by brain-derived neurotrophic factor in rats with cerebral ischaemia. J Int Med Res. 2011;39:488-498.

34. Toyama K, Honmou O, Harada K, et al. Therapeutic benefits of angiogenetic gene-modified human mesenchymal stem cells after cerebral ischemia. Exp Neurol. 2009;216:47-55.

35. Takahashi K, Okita K, Nakagawa M, Yamanaka S. Induction of pluripotent stem cells from fibroblast cultures. Nat Protoc. 2007;2:3081-3089.

36. Takahashi K, Tanabe K, Ohnuki M, et al. Induction of pluripotent stem cells from adult human fibroblasts by defined factors. Cell. 2007;131:861-872.

37. Ghosh Z, Wilson KD, Wu Y, Hu S, Quertermous T, Wu JC. Persistent donor cell gene expression among human induced pluripotent stem cells contributes to differences with human embryonic stem cells. PLOS ONE. 2010;5:e8975.

38. Hu BY, Zhang SC. Directed differentiation of neural-stem cells and subtypespecific neurons from hESCs. Methods Mol Biol. 2010;636:123-137. 